RESPIRATORY INFECTION

\title{
Genotypic variation in Pneumocystis jirovecii isolates in Britain
}

\author{
R F Miller, A R Lindley, A Copas, H E Ambrose, R J O Davies, A E Wakefield†
}

Thorax 2005;60:679-682. doi: 10.1136/thx.2004.039818

See end of article for authors' affiliations ....................

Correspondence to: Dr R F Miller, Centre for Sexual Health and HIV Research, Department of Primary Care and Population Sciences, Royal Free and University College Medical School, University College London, Mortimer Market Centre, London WCIE 6AU, UK; rmiller@gum.ucl.ac.uk

Received

24 December 2004

Accepted 15 May 2005

\begin{abstract}
Background: Pneumocystis jirovecii is the cause of Pneumocystis pneumonia (PCP) in immunosuppressed humans. Asymptomatic colonisation with $P$ jirovecii may occur in patients with minor immunosuppression or chronic lung disease. The aim of this study was to describe the molecular epidemiology of $P$ jirovecii in Britain over a period of 12.5 years.

Methods: Between January 1989 and July 2001161 samples of $P$ jirovecii were obtained from patients with PCP $(n=119)$, patients colonised by $P$ jirovecii $(n=35)$, and from air spora $(n=6)$. Genotyping of samples was performed at the mitochondrial large subunit rRNA (mt LSU rRNA).

Results: Genotype 1 (38\%) was the most frequently identified genotype: genotypes $2(26.6 \%), 3(20.3 \%)$, and $4(5 \%)$ were less common. Mixed infection (more than one genotype) was identified in 10\% of samples. While genotype 1 was the most frequently detected type in both patients with PCP and those colonised by $P$ jirovecii (38\% and $42 \%$, respectively), these groups differed in the relatively lower rate of detection of genotype $4(2 \% v 17 \%)$ and the higher detection of mixed infection in those with PCP $13 \% v$ $3 \%)$. Detection of specific genotypes of $P$ jirovecii was associated with the patient's place of residence $(p=0.02)$. There was no association between specific genotypes and severity of PCP as measured by arterial oxygen tension $(p=0.3)$.

Conclusions: The evidence of clustering of specific genotypes with patient's postcode of residence is consistent with the hypothesis of person to person transmission of $P$ jirovecii via the airborne route. The lack of association between specific mt LSU rRNA genotypes and severity of PCP suggests that this locus is not implicated in the virulence of the organism.
\end{abstract}

$\mathrm{T}$ he organism Pneumocystis jirovecii (previously called Pneumocystis carinii $\mathrm{f} \mathrm{sp}$ hominis) is the cause of Pneumocystis pneumonia (PCP) in patients immunosuppressed by HIV infection, malignancy, transplantation, and therapeutic immunosuppression. ${ }^{12}$ Among HIV infected patients in the developed world, there has been a marked reduction in the incidence of PCP as a result of the use of specific prophylaxis and of highly active antiretroviral therapy (HAART). ${ }^{3}$ However, PCP remains a common opportunistic infection, particularly among those who are unaware of their HIV serostatus at presentation or who are intolerant of or non-adherent with HAART and/or prophylaxis. ${ }^{4}$ In the developing world, HIV infected patients without access to prophylaxis and HAART remain susceptible to PCP. ${ }^{56}$ There is increasing evidence that $P$ jirovecii may also colonise the lungs of asymptomatic individuals with minor immunosuppression induced by $\mathrm{HIV},{ }^{78}$ malignancy, ${ }^{9}$ or long term receipt of glucocorticoids, ${ }^{10}$ immune competent persons with primary chronic lung disease, ${ }^{11-13}$ and pregnant women. ${ }^{14}$ It is hypothesised that these groups of individuals may act as a reservoir for human infection. ${ }^{8}{ }^{15}$

Study of the basic biology and epidemiology of $P$ jirovecii has been hampered by the lack of a reliable method for culture. Differences among isolates of $P$ jirovecii have been described using analysis of genetic polymorphisms at several loci. ${ }^{5-17}$ These differences have been used in studies of the epidemiology of $P$ jirovecii infection in the United States, Europe, and in Africa. ${ }^{5111618}$ There are few data on the epidemiology of $P$ jirovecii in Britain. ${ }^{19}$ The aim of this study was to describe the molecular epidemiology of $P$ jirovecii in Britain over a 12 year period. Genotyping was carried out at the mitochondrial large subunit rRNA (mt LSU rRNA). Samples were obtained from patients immunosuppressed by
HIV or other causes who had PCP, from patients colonised by $P$ jirovecii, and from samples of air spora.

\section{METHODS Samples}

Between January 1989 and July 2001, 119 bronchoscopic alveolar lavage (BAL) fluid samples were obtained from 116 patients ( 109 men) with PCP (defined by typical clinical and radiological presentation, identification of $P$ jirovecii cysts in BAL fluid by Grocott's methenamine silver staining, and by response to specific anti-pneumocystis therapy $\left.{ }^{20}\right)$. Of the 116 patients ( 115 adults), 110 were HIV infected with CD4 counts ranging from 10 to 400 cells/ $\mu$ l (median 60); six were not HIV infected and were immunosuppressed by malignancy $(n=5)$ or by immunosuppressive therapy for vasculitis $(n=1)$. Three patients with PCP underwent a second bronchoscopic examination because of failure to respond to specific treatment; the interval between bronchoscopies was 1723 days. In patients with PCP the following information was recorded from case note review: date of diagnosis of PCP, postcode of residence, admission oxygenation $\left(\mathrm{PaO}_{2}\right.$ breathing room air), and outcome.

Thirty six BAL fluid samples were from adult patients who did not have PCP and who were colonised with $P$ jirovecii. Nineteen of these patients were HIV infected men with CD4 counts ranging from 10 to 370 cells/ $\mu \mathrm{l}$ (median 20). All patients had alternative diagnoses, as defined previously; ${ }^{8}$ bacterial pneumonia in seven, pulmonary Kaposi sarcoma in five, cytomegalovirus pneumonitis in three, and Mycobacterium avium-intracellulare in three. Seventeen patients (14 men) were not HIV infected; their clinical details have been described

${ }^{\dagger}$ Deceased. 
elsewhere. ${ }^{10}$ Six samples of $P$ jirovecii were obtained from air samples collected from spore traps situated in a rural environment, as previously reported. ${ }^{21}$ Thus, a total of 161 isolates of $P$ jirovecii were studied. All patients undergoing bronchoscopy gave informed written consent and the study was performed within the guidelines of the Middlesex Hospital and Central Oxford Research Ethics Committees.

At the time of the diagnostic bronchoscopy an aliquot of BAL fluid was frozen at $-20^{\circ} \mathrm{C}$ and subsequently transferred to the Weatherall Institute of Molecular Medicine, Oxford, for analysis. The BAL fluid samples were coded and analyses were carried out blind to the patients' details.

\section{DNA extraction and amplification}

$P$ jirovecii DNA was extracted from BAL fluid samples as previously described. ${ }^{82-24}$ DNA amplification in patients with PCP was done using single round polymerase chain reaction (PCR) with primers pAZ102-H and pAZ102-E. In patients who were colonised with $P$ jirovecii and for air samples nested PCR was carried out. In the first round conditions were as above, and in the second round the primers pAZ102-X and pAZ102-Y were used. ${ }^{82-24}$ Extreme caution was taken to prevent cross contamination of samples. For example, DNA extraction was performed in a separate room and PCR amplification and DNA sequencing were carried out in different areas of the laboratory. At each stage handling of samples was done in a laminar flow cabinet and a new set of disposable-tip micropipettes, tubes, and reagents was used for each experiment. In each DNA extraction and PCR amplification experiment negative controls (ultrapurified distilled water instead of $P$ jirovecii DNA) were included. As a positive control, $P$ jirovecii DNA from a patient with PCP was used in each PCR amplification experiment.

\section{Sequencing of $\boldsymbol{P}$ iirovecii}

Amplification products were purified and either cloned and sequenced, or sequenced directly, as previously described. ${ }^{5} 822$ DNA sequence data were analysed either with Chromas 1.62 software (Technelysium, Pty, Tewantin, Australia) and University of Wisconsin Computer Group software version 10.1 (Genetics Computer Group, Wisconsin, USA), or with Gap4 of the Staden package (MRC Rosalind Franklin Centre for Genomics Research, Cambridge, UK). Genotypes of $P$ jirovecii were distinguished by identifying polymorphisms at position 85 and 248 of the mt LSU rRNA and were numbered using the method described by Beard et al. ${ }^{16}$

\section{Statistical analysis}

The $\chi^{2}$ test and the analysis of variance $F$ test were used for unadjusted tests of association between genotype of $P$ jirovecii and categorical and continuous factors, respectively. Multinomial logistic regression with genotype as the outcome was used to simultaneously examine the association of multiple factors. Mixed genotypes were considered as one category for analysis. In the three patients with PCP who underwent repeat sampling, only the first sample was used for genotypic analysis. Area of residence was assigned from postcodes into six categories: North, South, East and West London, Oxford region and "other". For associations with time a linear trend was tested, and "clustering by time" was tested by grouping time based on frequencies as 1989-91, 1992-3, 1994-6, and 1997-2001. A p value of $<0.05$ was considered significant. Statistical analyses were performed using STATA Version 8.

\section{RESULTS}

Genotype 1 (38\%) was the most common type identified; genotypes $2(26.6 \%), 3(20.3 \%)$, and $4(5 \%)$ were less common. In $10 \%$ of samples co-infection with two genotypes (mixed infection) was detected (table 1).

Among the limited number of air spora samples, genotype $2(50 \%)$ was most frequently identified (fig 1), whereas mixed infection and genotype 4 were not identified. While genotype 1 was the most frequently detected type among both patients with PCP and in those colonised by $P$ jirovecii (38\% and $42 \%$ respectively, table 1), these two groups differed significantly $(p=0.004)$ in their genotype distribution due to the relatively lower detection of genotype 4 ( $2 \% \mathrm{v}$ $17 \%)$ and higher detection of mixed infection among those with PCP $(13 \% \vee 3 \%)$. The difference remained significant after adjusting for year of sample.

In patients with PCP, analysis of the association between detection of specific genotypes and year of diagnosis of PCP showed little association $(p=0.31$, linear trend and $p=0.12$, test for "time clustering"). Detection of specific genotypes of $P$ jirovecii was associated with patient's place of residence $(\mathrm{p}=0.02$, fig 1$)$. For example, among 28 patient samples from South London, 17 (61\%) were genotype 1, but only one (4\%) was genotype 3 and two (7\%) were mixed infection. By contrast, of 36 samples from North London, eight (22\%) were genotype 1 , nine $(25 \%)$ were genotype 3 , and nine $(25 \%)$ were mixed infection.

All patients with PCP survived; there was no association between specific genotypes of $P$ jirovecii and severity of PCP as measured by $\mathrm{PaO}_{2}(\mathrm{p}=0.30)$. In the three patients with PCP who underwent a repeat bronchoscopy, the same genotype of $P$ jirovecii (type 2 in two patients and type 3 in one) was identified in the first and second BAL fluid samples. Genotype 1 was detected in the child with PCP.

\section{DISCUSSION}

This study from Britain is the first to describe genotyping of isolates of $P$ jirovecii obtained contemporaneously from

\begin{tabular}{|c|c|c|c|c|c|}
\hline \multirow[b]{2}{*}{ Genotype } & \multicolumn{3}{|c|}{ Sample } & \multirow[b]{2}{*}{ Total } & \multirow[b]{2}{*}{$\%$} \\
\hline & $\overline{P C P}$ & Colonised & Air spora & & \\
\hline 1 & 44 & 15 & 1 & 60 & 38 \\
\hline 2 & 30 & 9 & 3 & 42 & 26.6 \\
\hline 3 & 25 & 5 & 2 & 32 & 20.3 \\
\hline 4 & 2 & 6 & 0 & 8 & 5.1 \\
\hline \multicolumn{6}{|l|}{ Mixed } \\
\hline $5(1+3)$ & 9 & 0 & 0 & 9 & 5.6 \\
\hline $6(1+2)$ & 3 & 0 & 0 & 3 & 1.9 \\
\hline $8(1+4)$ & 2 & 1 & 0 & 3 & 1.9 \\
\hline $9(2+3)$ & 1 & 0 & 0 & 1 & 0.6 \\
\hline Total & 116 & 36 & 6 & 158 & 100 \\
\hline
\end{tabular}




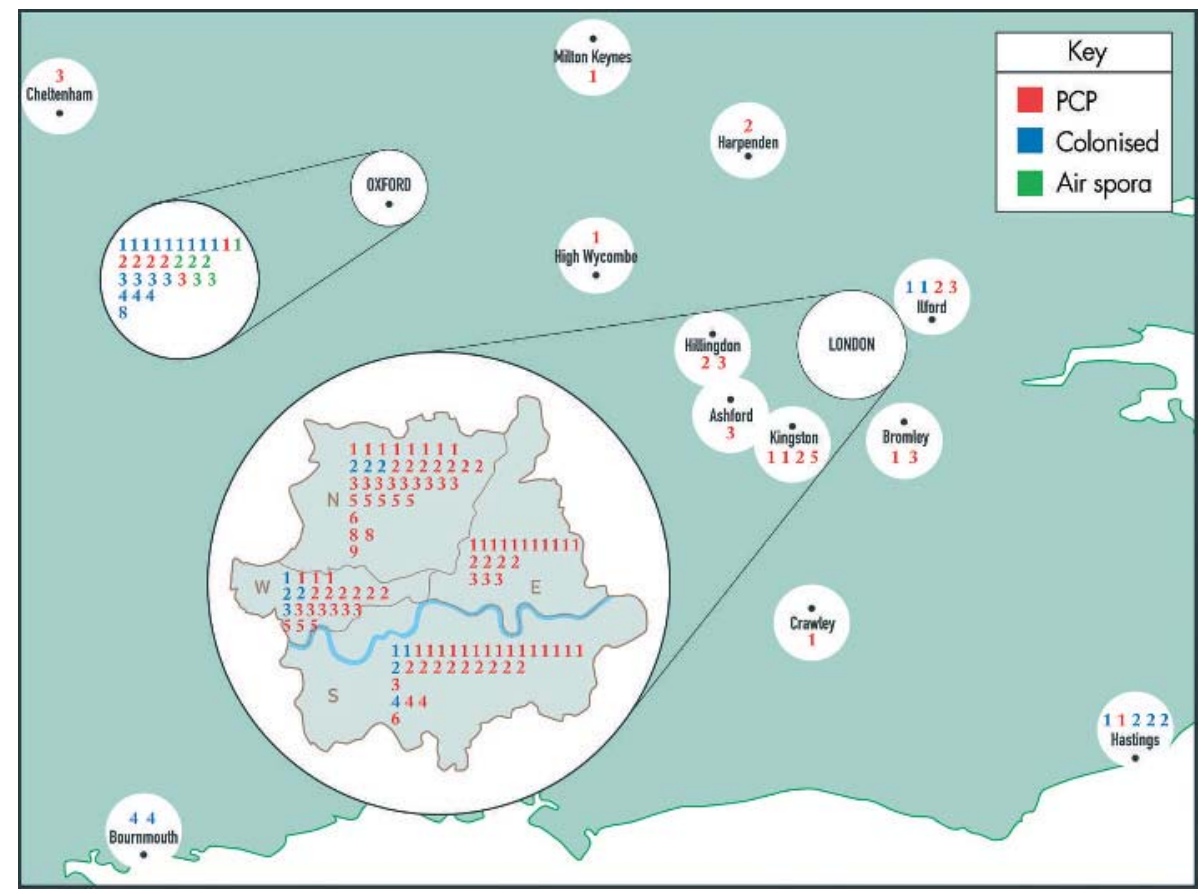

Figure 1 Map showing the geographical location of isolates of genotypes of Pneumocystis jirovecii in samples from patients with PCP ( $n=116$ ), patients colonised with $P$ jirovecii $(n=36)$, and from air spora $(n=6)$.

patients with PCP, from patients colonised by $P$ jirovecii, and from air spora. Analysis was performed at the mt LSU rRNA, which is an informative locus not under selective pressure from sulpha drug exposure that has been used previously in epidemiological studies. ${ }^{11}{ }^{16}$ Of five different genotypes previously described at this locus, four were identified in this study. Use of single round PCR enabled detection of $P$ jirovecii DNA in respiratory samples from patients with PCP, whereas nested PCR was needed to detect $P$ jirovecii DNA in samples from those colonised by $P$ jirovecii and in air spora, reflecting the lower levels of DNA in these samples. ${ }^{8}$

Genotype 1 was the commonest genotype identified in this study, accounting for $38 \%$ of isolates. Similar results have been reported in a multicentre study of 324 patients with PCP from the United States (genotype 1 frequency $38 \%)^{16}$ and a study of 79 isolates from patients with PCP and patients colonised by $P$ jirovecii from Seville, Spain (genotype 1 frequency $49.3 \%) .{ }^{11}$ By contrast, a preliminary study of 14 patients with PCP from Zimbabwe reported genotype 1 in only $28.6 \%$; genotype 3 was the most frequently identified (57\%). ${ }^{5}$ In the present study genotype 3 accounted for $20.3 \%$ of isolates, which contrasts with results from the USA $(9.3 \%$ genotype 3$)^{16}$ and from Seville (36.7\% genotype 3$) .{ }^{11}$ Mixed infections ( $>1$ genotype in a sample) were identified in $10 \%$ of isolates in this study; a similar rate of detection was reported in the study from USA $(10.2 \%) .{ }^{16}$ This rate is almost three times greater than the rate of detection (3.7\%) of mixed infection in the Spanish study. ${ }^{11}$ The absence of change in the frequency of detection of different genotypes over the 12.5 years of the study suggests stability of this genetic locus and demonstrates its usefulness in longitudinal epidemiological studies of $P$ jirovecii infection.

The distribution of genotypes in samples from patients with PCP and those colonised by $P$ jirovecii differed significantly but also showed some similarities-for example, genotype 1 being most common. The distribution of genotypes in air spora was somewhat different, but the number of samples was limited. These genotypic data are consistent with the hypothesis that Pneumocystis is transmitted via the airborne route from person to person. Further evidence from this study-which supports the hypothesis that PCP arises by de novo acquisition of infection-comes from the observation that, in patients with PCP, specific genotypes of $P$ jirovecii were associated with residential postcode. Geographical clustering of cases of PCP by postcode (zip code) has previously been described in studies from Cincinnati ${ }^{25}$ and San Francisco. ${ }^{26} 27$ Recent acquisition of infection may also be inferred from the finding that allelic variation patterns in isolates of $P$ jirovecii from patients with PCP are correlated with patient's place of diagnosis and not their place of birth. ${ }^{16}$

This study found no correlation between specific genotypes of $P$ jirovecii at the mt LSU rRNA locus and severity of PCP, as judged by the patient's admission $\mathrm{PaO}_{2}$ (breathing room air). This observation corroborates the results of another study that showed a lack of association between severity of PCP (using admission $\mathrm{PaO}_{2}$ ) and specific genotypes of $P$ jirovecii at the internal transcribed spacer (ITS) regions of rRNA. ${ }^{28}$ These data suggest that the genotype of $P$ jirovecii at both the $\mathrm{mt}$ LSU rRNA and ITS loci does not influence PCP disease severity.

In the three patients who underwent repeat BAL during an episode of PCP because of failure to respond to specific treatment, there was no change in genotype at the mt LSU rRNA which suggests that $P$ jirovecii does not mutate in the human host during an episode of PCP. Our observations confirm previous reports of four patients in the USA ${ }^{29}$ and 10 in France ${ }^{30}$ in whom no change in genotype was observed in patients with PCP who underwent repeat BAL after an interval of 7-30 days.

This study has shown the usefulness of genotyping at the mt LSU rRNA for describing the molecular epidemiology (particularly in longitudinal studies) of isolates of $P$ jirovecii from patients with PCP, those colonised with the organism, and in samples of air spora. The genotype distribution in isolates from all three groups had similarities consistent with the concept of person to person transmission via the airborne route. An association between patient's postcode of residence 
and specific genotypes further supports the concept of "recent" acquisition of infection. The lack of association between disease severity and genotype suggests that other loci are involved in determining the virulence of this organism and indicates that prospective studies are needed to address these issues.

\section{ACKNOWLEDGEMENTS}

The authors thank Mary Deadman, Department of Paediatrics, Weatherall Institute of Molecular Medicine, University of Oxford, for facilitating data collection.

\section{Authors' affiliations}

R F Miller, A Copas, Centre for Sexual Health and HIV Research, Department of Primary Care and Population Sciences, Royal Free and University College Medical School, University College London, London WICE 6AU, UK

A R Lindley, H E Ambrose, A E Wakefield, Molecular Infectious Diseases Group, Department of Paediatrics, Weatherall Institute of Molecular Medicine, University of Oxford, Oxford OX3 9DU, UK

R J O Davies, Oxford Centre for Respiratory Medicine, Churchill Hospital, Oxford Radcliffe NHS Trust, Oxford OX3 7ப, UK

Financial support: Royal Society (AEW), Wellcome Trust (AEW), Medical Research Council (HEA), Fifth Framework Programme of the European Commission contract QLK2-CT-2000-01369 (ARL, RFM and AEW), Camden PCT (RFM)

Competing interests: RFM is Editor of Sexually Transmitted Infections, published by the BMJ Publishing Group

\section{REFERENCES}

1 Stringer JR, Beard CB, Miller RF, et al. A new name (Pneumocystis jiroveci) for Pneumocystis from humans. Emerg Infect Dis 2002;8:891-6.

2 Morris A, Lundgren JD, Masur $\mathrm{H}$, et al. Current epidemiology of Pneumocystis pneumonia. Emerg Infect Dis 2004;10:1713-20.

3 Kaplan JE, Hanson D, Dworkin MS, et al. Epidemiology of human immunodeficiency virus-associated opportunistic infections in the United States in the era of highly active antiretroviral therapy. Clin Infect Dis 2000;30(Suppl 1):S5-14.

4 Lundberg BE, Davidson AJ, Burnam WJ. Epidemiology of Pneumocystis carinii pneumonia in an era of effective prophylaxis: relative contribution of non adherence and drug failure. AIDS 2000;14:2559-66.

5 Miller RF, Lindley AR, Ambrose HE, et al. Genotypes of Pneumocystis jiroveci isolates obtained in Harare, Zimbabwe, and London, United Kingdom. Antimicrob Agents Chemother 2003;47:3979-81.

6 Zar HJ, Alvarez-Martinez MJ, Harrison A, et al. Prevalence of dihydropteroate synthase mutants in HIV-infected South African children with Pneumocystis jiroveci pneumonia. Clin Infect Dis 2004;39:1047-51.

7 Nevez G Raccurt C, Jounieaux V, et al. Pneumocystosis versus Pneumocystis colonization in HIV-negative and HIV-positive patients. AIDS 1999;13:535-6.

8 Wakefield AE, Lindley AR, Ambrose HE, et al. Limited asymptomatic carriage of Pneumocystis jiroveci in human immunodeficiency virus-infected patients. $J$ Infect Dis 2003;187:901-8.
9 Henson JW Jalai JK, Walker RW et al. Pneumocystis carinii pneumonia in patients with brain tumors. Arch Neurol 1991;48:406-9.

10 Maskell NA, Waine DJ, Lindley A, et al. Asymptomatic carriage of Pneumocystis jiroveci in subjects undergoing bronchoscopy: a prospective study. Thorax 2003:58:594-7.

11 Montes-Cano MA, de la Horra C, Martin-Juan J, et al. Pneumocystis jiroveci genotypes in the Spanish population. Clin Infect Dis 2004;39:123-8.

12 Sing A, Roggenkamp A, Autenreith IB, et al. Pneumocystis carinii carriage in immunocompetent patients with primary pulmonary disorders as detected by single or nested PCR. J Clin Microbiol 1999;37:3409-10.

13 Armbruster C, Hassl A, Kriwanek S. Pneumocystis carinii colonization in the absence of immune suppression. Scand J Infect Dis 1997;29:591-3.

14 Vargas SL, Ponce CA, Sanchez LA, et al. Pregnancy and asymptomatic carriage of Pneumocystis jiroveci. Emerg Infect Dis 2003;9:605-6.

15 Beard CB, Roux $P, N e v e z G$, et al. Strain typing methods and molecular epidemiology of Pneumocystis pneumonia. Emerg Infect Dis 2004; 10:1729-35

16 Beard CB, Carter JL, Keely SP, et al. Genetic variation in Pneumocystis carinii isolates from different geographic regions: implications for transmission. Emerg Infect Dis 2000;6:265-72.

17 Lee CH, Helweg-Larsen J, Tang X, et al. Update of Pneumocystis carinii f. sp. hominis typing based on nucleotide sequence variation in internal transcribed spacer regions of rRNA genes. J Clin Microbiol 1998;36:734-41.

18 Robberts FJ, Liebovitz LD, Chalkley L. Genotyping and coalescent phylogenetic analysis of Pneumocystis jiroveci from South Africa. J Clin Microbiol 2004;42:1505-20.

19 Miller RF, Wakefield AE. Genotypes of Pneumocystis carinii and severity of pneumonia. Lancet 1999:353:2039-40.

20 Miller RF, Millar AB, Weller IVD, et al. Empirical treatment without bronchoscopy for Pneumocystis carinii pneumonia in the acquired immunodeficiency syndrome. Thorax 1989;44:559-64.

21 Wakefield AE. DNA sequences identical to Pneumocystis carinii f. sp. carinii and Pneumocystis carinii f. sp. hominis in samples of air spora. J Clin Microbiol 1996;34:1754-9.

22 Tsolaki AG, Beckers P, Wakefield AE. Pre-AIDS era isolates of Pneumocystis carinii f. sp. hominis: high genotypic similarity with contemporary isolates, J Clin Microbiol 1998;36:90-3.

23 Wakefield AE, Pixley FJ, Banerii S, et al. Detection of Pneumocystis carinii with DNA amplification. Lancet 1990;336:451-3.

24 Miller RF, Ambrose HE, Wakefield AE. Pneumocystis carinii f. sp. hominis DNA in healthcare workers in contact with patients with Pneumocystis carinii pneumonia. J Clin Microbiol 2001;39:3877-82.

25 Dohn MN, White ML, Vigdorth EM, et al. Geographic clustering of Pneumocystis carinii pneumonia in patients with HIV infection. Am J Respir Crit Care Med 2000;162:1617-21

26 Morris AM, Swanson M, Ha H, et al. Geographic distribution of human immunodeficiency virus-associated Pneumocystis carinii pneumonia in San Francisco. Am J Respir Crit Care Med 2000;162:1622-6.

27 Huang L, Beard CB, Creasman J, et al. Sulfa or sulfone prophylaxis and geographic region predict mutations in the Pneumocystis carinii dihydropteroate synthase gene. J Infect Dis 2000;182:1192-8.

28 Helweg-Larsen J, Lee C-H, Jin S, et al. Clinical correlation of variations in the internal transcribed spacer regions of rRNA genes in Pneumocystis carinii $f$ sp. hominis. AIDS 2001;15:451-9.

29 Keely SP, Stringer JR, Baughman RP, et al. Genetic variation among Pneumocystis carinii hominis isolates in recurrent pneumocystis. J Infect Dis 1995; 172:595-8.

30 Latouche S, Poirot J-L, Bernard C, et al. Study of internal transcribed spacer and mitochondrial large subunit genes of Pneumocystis carinii hominis isolated by repeated bronchoalveolar lavage from human immunodeficiency virus-infected patients during one or several episodes of pneumonia. J Clin Microbiol 1997;35:1687-90. 